\title{
Cardiac output, pulmonary artery pressure, and patent ductus arteriosus during therapeutic cooling after global hypoxia-ischaemia
}

\author{
D Fugelseth, S Satas, P A Steen, M Thoresen
}

Arch Dis Child Fetal Neonatal Ed 2003;88:F223-F228

See end of article for authors' affiliations

\section{Correspondence to:} Dr Thoresen, Department of Child Health, St Michael Hospital, Southwell Street, Bristol BS2 8EG, UK; marianne.thoresen@ bristol.ac.uk

Accepted 27 August 2002

\begin{abstract}
Objective: To assess by Doppler echocardiography the effects of 24 hours of whole body mild hypothermia compared with normothermia on cardiac output (CO), pulmonary artery pressure (PAP), and the presence of a persistent ductus arteriosus (PDA) after a global hypoxic-ischaemic insult in unsedated newborn animals.

Design: Thirty five pigs (mean (SD) age 26.6 (12.1) hours and weight $1.6(0.3) \mathrm{kg}$ ) were anaesthetised with halothane, mechanically ventilated, and subjected to a 45 minute global hypoxic-ischaemic insult. At the end of hypoxia, halothane was stopped; the pigs were randomised to either normathermia $\left(39^{\circ} \mathrm{C}\right)$ or hypothermia $\left(35^{\circ} \mathrm{C}\right)$ for 24 hours. Rewarming was carried out for $24-30$ hours followed by 42 hours of normothermia. Unanaesthetised pigs were examined with a VingMed CFM 750 ultrasound scanner before and 3, 24, 30, and 48 hours after the hypoxic-ischaemic insult. Aortic valve diameter, forward peak flow velocities across the four valves, and the occurrence of a PDA were measured. Tricuspid regurgitation (TR) velocity was used to estimate the PAP. Stroke volume was calculated from the aortic flow.

Results: Twelve animals (seven normothermic, five hypothermic) had a PDA on one or more examinations, which showed no association with cooling or severity of insult. There were no differences in stroke volume or TR velocity between the hypothermic and normothermic animals at any time point after the insult. CO was, however, $45 \%$ lower at the end of cooling in the subgroup of hypothermic pigs that had received a severe insult compared with the pigs with mild and moderate insults. $C O$ and TR velocity were transiently increased three hours after the insult: $0.38(0.08) v 0.42(0.08)$ litres $/ \mathrm{min} / \mathrm{kg}$ $(p=0.007)$ for CO; $3.0(0.42) \vee 3.4(0.43) \mathrm{m} / \mathrm{s}(\mathrm{p}<0.0001)$ for TR velocity (values are mean (SD)). Conclusions: The introduction of mild hypothermia while the pigs were unsedated did not affect the incidence of PDA nor did it lead to any changes in MABP or PAP. Stroke volume was also unaffected by temperature, but hypothermic piglets subjected to a severe hypoxic-ischaemic insult had reduced $\mathrm{CO}$ because the heart rate was lower. Global hypoxia-ischaemia leads to similar transient increases in $\mathrm{CO}$ and estimated PAP in unsedated normothermic and hypothermic pigs. There were no signs of metabolic compromise in any subgroup, suggesting that 24 hours of mild hypothermia had no adverse cardiovascular effect.
\end{abstract}

M ild hypothermia is currently one of the most promising clinically feasible "neural rescue therapies" for full term infants with hypoxic-ischaemic encephalopathy. ${ }^{1}$ In cardiac surgery, severe to moderate $\left(20-32^{\circ} \mathrm{C}\right)$ hypothermia during the operation has been associated with adverse cardiovascular effects. ${ }^{2}$ Less attention has been given to the possible adverse physiological effects of mild-that is, rectal temperature of $33-35^{\circ} \mathrm{C}$-hypothermia. Recently we showed that hypothermia after hypoxia in unsedated piglets increased cortisol levels significantly, and cortisol is a good marker of stress in pigs. ${ }^{3}$ In our pilot study of cardiovascular changes during cooling and rewarming, involving nine unsedated infants with neonatal hypoxicischaemic encephalopathy, we observed that mean arterial blood pressure (MABP) increased by a median of $10 \mathrm{~mm} \mathrm{Hg}$ during the start of cooling and fell by a median of $8 \mathrm{~mm} \mathrm{Hg}$ on rewarming. Heart rate (HR) decreased by a median of 34 beats/min and increased by a median of 32 beats/min on rewarming. ${ }^{4}$

The current knowledge on the effect of hypothermia on the cardiovascular system has mostly been obtained from fully anaesthetised or heavily sedated adult subjects. This differs from the clinical practice in neonates who are often ventilated with little or no sedation. ${ }^{5}$ Neonates who are hypoxic after birth may reopen their ductus arteriosus. We do not know whether cooling affects the patency of the duct. Also hypothermic neonates are thought to develop pulmonary hypertension more easily. There are continuing clinical trials of mild hypothermia $\left(33-34^{\circ} \mathrm{C}\right.$ core temperature) lasting for 72 hours in infants with severe hypoxic-ischaemic encephalopathy. We therefore wanted to examine the effect of temperature on cardiac output (CO), pulmonary artery pressure (PAP), and the patency of the ductus arteriosus (PDA) in the absence of anaesthesia after hypoxic-ischaemic encephalopathy, in a newborn piglet model shown to develop neurological and pathological changes similar to the human baby. ${ }^{6}$ Echocardiography is the main tool used in neonatal intensive units today to study cardiovascular effects in neonates as well as adults. We aimed to study the circulatory consequences over time in an animal survival model that most closely resembles the clinical setting with regard to management and monitoring after a hypoxic-ischaemic insult.

Abbreviations: $M A B P$, mean arterial blood pressure; $H R$, heart rate; CO, cardiac output; PAP, pulmonary artery pressure; PDA, patient ductus arteriosus; $\mathrm{EEG}$, electroencephalogram; $\mathrm{FIO}_{2}$, fractional inspired oxygen; $T R$, tricuspid regurgitation 


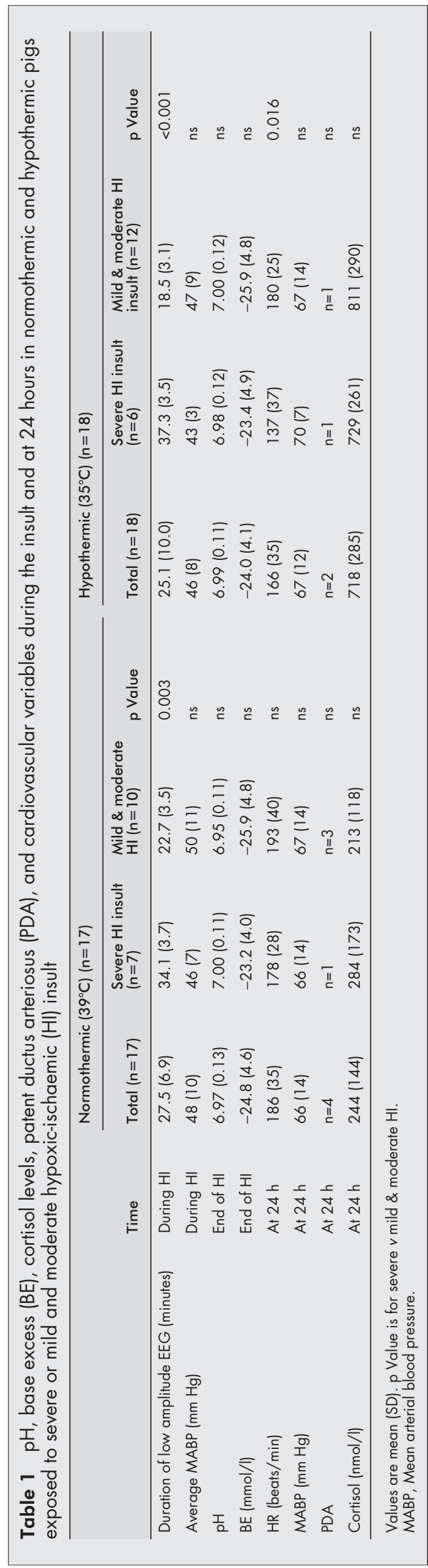

The combination of the physiological consequences of hypoxia-ischaemia and the circulatory transition from the fetus to the newborn state may well lead to a different response in newborns compared with the anaesthetised adult human. Therefore one cannot safely extrapolate from the results obtained in anaesthetised adults undergoing therapeutic hypothermia.

We have previously described a standardised method of two dimensional Doppler echocardiography and the normal ranges for cardiac functions in unsedated newborn pigs. ${ }^{7}$ The aim of the present study was also to assess by Doppler echocardiography the effects on CO, PAP, and the presence of PDA of 24 hours of mild hypothermia without sedation as compared with normothermia, after a global hypoxicischaemic insult in newborn piglets.

\section{METHODS}

The Norwegian Council for Animal Research approved the study protocol. Thirty five Landrace newborn (where newborn is defined as up to 48 hours of age) pigs (26.6 (12.1) hours old weighing $1.6(0.3) \mathrm{kg}$ (mean (SD)) were included in the study, and planned survival was 72 hours. They remained with the sow until less than three hours before the experiment started. Four pigs died prematurely from complications secondary to the hypoxic-ischaemic insult: one from the hypothermic group at 24-30 hours, and two from the hypothermic group and one from the normothermic group at 30-48 hours. Cardiovascular data obtained from these animals until they died were included in the results.

The piglets were anaesthetised with halothane $(3 \%$ induction followed by $0.6-0.8 \%$ maintenance), intubated, and
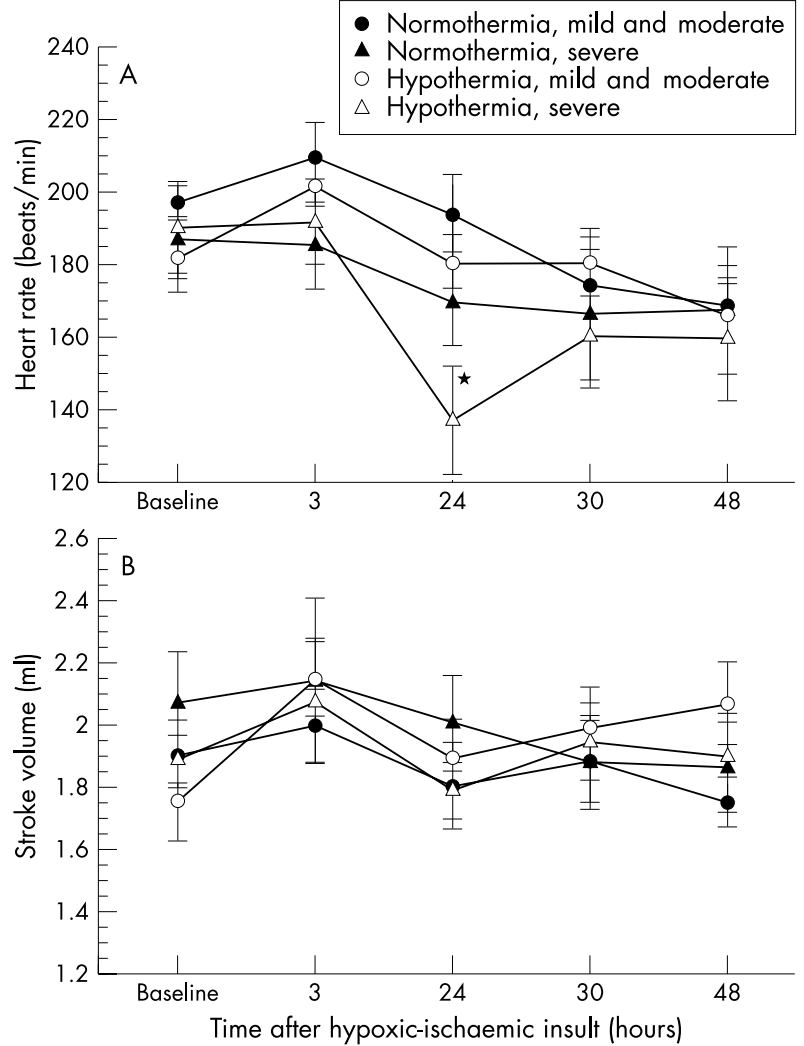

Figure 1 (A) Changes in and the effect of the severity of the hypoxic-ischaemic insult (mild and moderate or severe) on heart rate in the normothermic and hypothermic groups. There is a significant reduction in hypothermic piglets with severe injury $\left({ }^{*} p<0.05\right)$. (B)

Changes in and the effect of the severity of the hypoxic-ischaemic insult (mild and moderate or severe) on stroke volume in the normothermic and hypothermic groups. The stroke volume is unchanged and similar in all groups. Values are mean (SEM). 

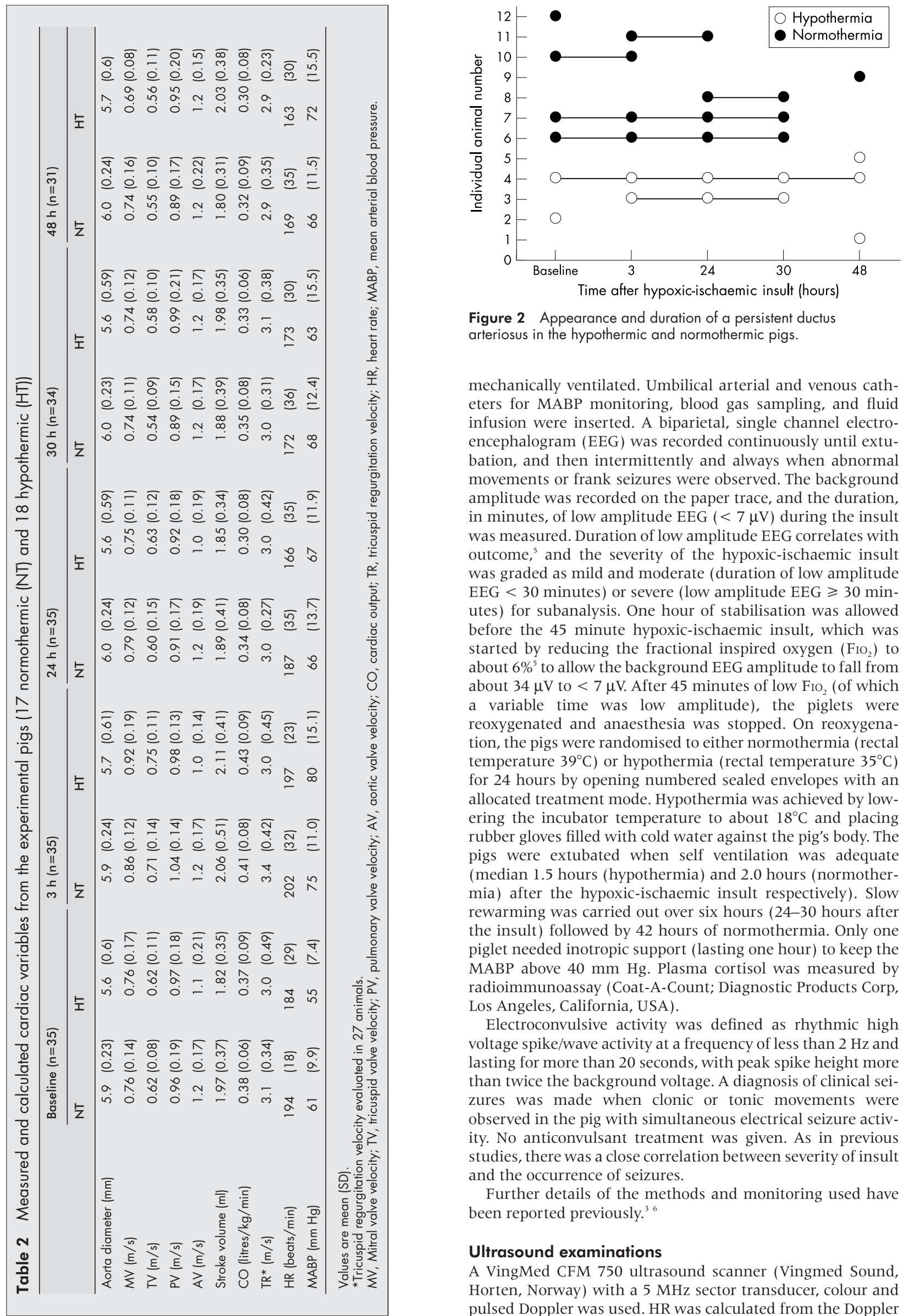

Figure 2 Appearance and duration of a persistent ductus arteriosus in the hypothermic and normothermic pigs.

mechanically ventilated. Umbilical arterial and venous catheters for MABP monitoring, blood gas sampling, and fluid infusion were inserted. A biparietal, single channel electroencephalogram (EEG) was recorded continuously until extubation, and then intermittently and always when abnormal movements or frank seizures were observed. The background amplitude was recorded on the paper trace, and the duration, in minutes, of low amplitude EEG $(<7 \mu \mathrm{V})$ during the insult was measured. Duration of low amplitude EEG correlates with outcome, ${ }^{5}$ and the severity of the hypoxic-ischaemic insult was graded as mild and moderate (duration of low amplitude EEG $<30$ minutes) or severe (low amplitude EEG $\geqslant 30$ minutes) for subanalysis. One hour of stabilisation was allowed before the 45 minute hypoxic-ischaemic insult, which was started by reducing the fractional inspired oxygen $\left(\mathrm{FiO}_{2}\right)$ to about $6 \%$ to allow the background EEG amplitude to fall from about $34 \mu \mathrm{V}$ to $<7 \mu \mathrm{V}$. After 45 minutes of low $\mathrm{FIO}_{2}$ (of which a variable time was low amplitude), the piglets were reoxygenated and anaesthesia was stopped. On reoxygenation, the pigs were randomised to either normothermia (rectal temperature $39^{\circ} \mathrm{C}$ ) or hypothermia (rectal temperature $35^{\circ} \mathrm{C}$ ) for 24 hours by opening numbered sealed envelopes with an allocated treatment mode. Hypothermia was achieved by lowering the incubator temperature to about $18^{\circ} \mathrm{C}$ and placing rubber gloves filled with cold water against the pig's body. The pigs were extubated when self ventilation was adequate (median 1.5 hours (hypothermia) and 2.0 hours (normothermia) after the hypoxic-ischaemic insult respectively). Slow rewarming was carried out over six hours (24-30 hours after the insult) followed by 42 hours of normothermia. Only one piglet needed inotropic support (lasting one hour) to keep the MABP above $40 \mathrm{~mm} \mathrm{Hg}$. Plasma cortisol was measured by radioimmunoassay (Coat-A-Count; Diagnostic Products Corp, Los Angeles, California, USA).

Electroconvulsive activity was defined as rhythmic high voltage spike/wave activity at a frequency of less than $2 \mathrm{~Hz}$ and lasting for more than 20 seconds, with peak spike height more than twice the background voltage. A diagnosis of clinical seizures was made when clonic or tonic movements were observed in the pig with simultaneous electrical seizure activity. No anticonvulsant treatment was given. As in previous studies, there was a close correlation between severity of insult and the occurrence of seizures.

Further details of the methods and monitoring used have been reported previously. ${ }^{36}$

\section{Ultrasound examinations}

A VingMed CFM 750 ultrasound scanner (Vingmed Sound, Horten, Norway) with a $5 \mathrm{MHz}$ sector transducer, colour and pulsed Doppler was used. HR was calculated from the Doppler 
flow tracings. The same investigator performed all cardiac examinations (DF) so that the data would not be subject to interobserver variability. It was not possible to blind the observer to treatment allocation of hypothermia. The recordings were stored on colour videotapes as well as black and white paper prints for analysis, and the treatment allocation was blinded on these. All ultrasound examinations were performed while the pigs were unsedated. They were lying in a left lateral, half supine position, ${ }^{7}$ often held by an assistant. The ultrasound examinations were performed before and three hours, 24 hours (end of hypothermia), 30 hours (end of rewarming), and 48 hours after the hypoxic-ischaemic insult.

From the low midcostal long and short axis views and a right sided parasternal long axis view, the cardiac morphology, the occurrence of a PDA, the aortic valve diameter, and the forward peak velocities across the four valves were measured using echocardiographs, Doppler ultrasound, and colour Doppler as previously described. ${ }^{7}$ The tricuspid regurgitation (TR) peak velocity signal was manually traced for calculation of the pressure gradient between the right ventricle and right atrium $(\mathrm{mm} \mathrm{Hg})$. For this calculation, the in built program in the CFM 750 applies the modified Bernoulli's equation. ${ }^{8}$ Systolic PAP is then equal to the systolic pressure difference calculated plus the right atrial pressure. ${ }^{9}$ The pressure in the right atrium is commonly assumed to be about $2 \mathrm{~mm} \mathrm{Hg} \cdot{ }^{10} \mathrm{CO}$ was calculated from the stroke volume (average mean velocity across the aortic root $\times$ vessel area) $\times$ HR. The aortic valve diameter of each pig was measured at the first examination, and these values were then used for all further calculations of the CO.

The cardiac morphology, aortic valve diameter, and the persistence of a ductus arteriosus (by Doppler and colour flow) were easily evaluated in all animals, ${ }^{7}$ as well as the velocities across the four valves and the CO. A full set of examinations of the TR velocity was obtained in only 27 pigs because of technical difficulties. The eight piglets (four hypothermic, four normothermic) not examined for TR velocity did not differ from the 27 examined with respect to severity of insult or MABP.

All measurements used in the final calculations for each pig were the mean values, automatically calculated in the machine, from three to five repeated selected sequential beats regarded as good quality measurements. For descriptive statistics, mean (SD) or median (total range) was used. For comparison between or within groups, parametric or nonparametric, unpaired or paired tests were used as appropriate. $\mathrm{p}=0.05$ was regarded as significant (two sided testing).

\section{RESULTS}

Body weight, age, sex, rectal temperature, and cardiovascular and biochemical variables at baseline were similar in the normothermic and hypothermic pigs. The characteristics of the hypoxic-ischaemic insult (duration of low amplitude EEG, average MABP, and end insult $\mathrm{pH}$ and base excess) were the same in normothermic and hypothermic animals (table 1). HR was significantly lower in the hypothermic piglets that had received a severe insult (fig 1A). Cortisol levels did not depend on the severity of the insult; however, they were fourfold higher in the hypothermic than the normothermic piglets (table 1). The occurrence of a PDA was low and no difference was observed between the two conditions.

Table 2 presents measured and calculated cardiac variables for the experimental pigs. There was no difference in any of the variables between the normothermic and hypothermic group. In fig $1 \mathrm{HR}$ and stroke volume are displayed according to the severity of the insult. In hypothermic pigs with a severe insult (low amplitude EEG $\geqslant 30$ minutes), HR was significantly lower at the end of hypothermia. The stroke volume was the same in all groups and did not change over time.

All animals had normal atrial and ventricular arrangements and connections. The hearts were structurally normal except

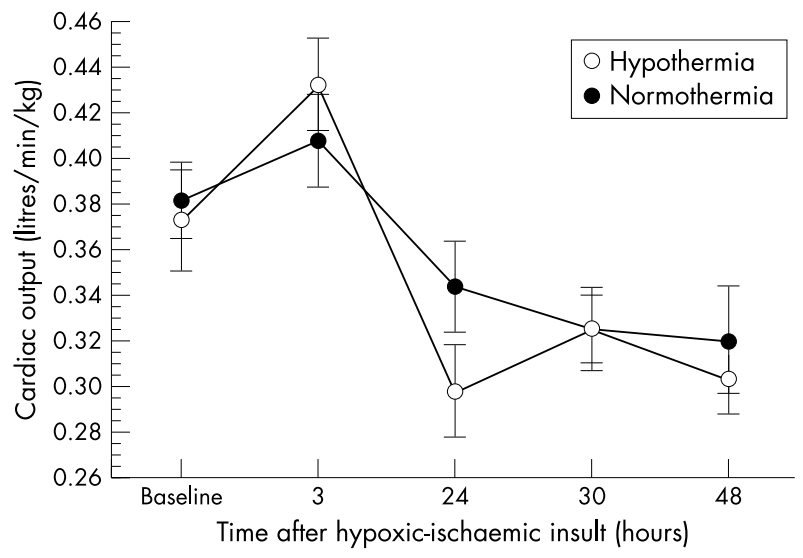

Figure 3 Cardiac output in the hypothermic and normothermic pigs throughout the experimental period. There is an increase from baseline to three hours after the hypoxic-ischaemic insult followed by a decrease between 3 hours and 24 hours. Values are mean (SEM).

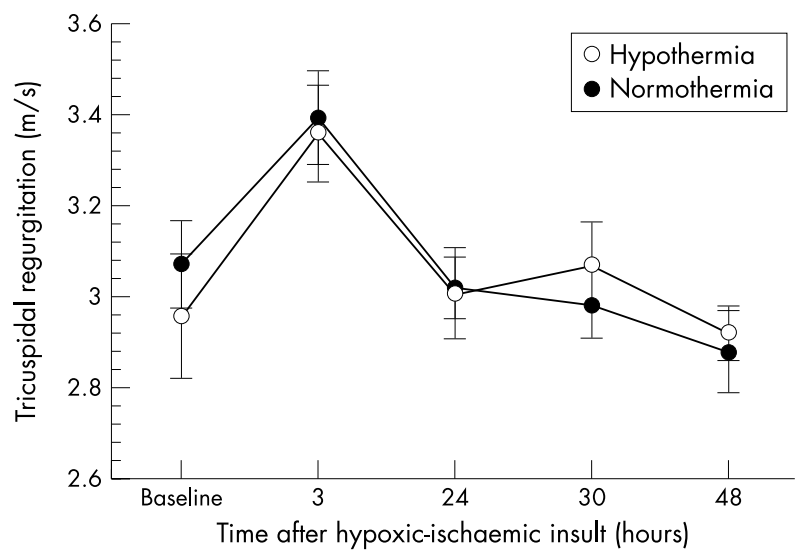

Figure 4 Time course of tricuspid regurgitation velocity. There is a transient increase from baseline to three hours after the insult in both normothermic and hypothermic animals. Values are mean (SEM).

in two pigs: one had a $5 \mathrm{~mm}$ atrial septal defect and one a small muscular ventricular septal defect. Twelve animals (seven normothermic, five hypothermic) had their ductus arteriosus open on one or more examinations. The appearance and duration of the open duct (fig 2) was similar in the two groups. Five animals (three hypothermic and three normothermic) developed PDA at different times after the hypoxicischaemic insult. Opening of the duct did not relate to whether the animals were cooled or not or the severity of the insult (table 1).

We found that CO had transiently (and similarly for normothermic and hypothermic pigs) increased three hours after the hypoxic-ischaemic insult: $0.38(0.08) v 0.42(0.08)$ litres $/ \mathrm{min} / \mathrm{kg}, \mathrm{p}=0.007$ (fig 3 ). There was a transient increase $(p<0.0001)$ in PAP estimated from the TR peak velocity three hours after the insult in all animals. From 24 hours onwards, the values were no different from baseline (fig 4). A PDA did not influence the PAP results.

\section{DISCUSSION}

In this study the animals were unsedated in the period after hypoxia, which is normal clinical practice for newborn infants. The hypothermic animals had high cortisol levels, and we have interpreted this as a sign of stress because of lack of sedation. Adults or children subjected to hypothermia during cardiac surgery, or as neuroprotection, have always been anaesthetised or sedated, and these patients invariably have reduced HR. We only found a reduction in HR in the subgroup of 
hypothermic animals that were the most severely damaged. These animals showed much less spontaneous activity than those moderately damaged. The stroke volume was similar in all groups and subgroups, therefore the observed reduction in CO was solely due to the reduced HR. Also, as no acidosis or hypotension was seen during hypothermia, CO was sufficient for metabolic needs. Previous studies have found that mild hypothermia $\left(33-35^{\circ} \mathrm{C}\right)$ increased the stroke volume; however, at temperatures $<32^{\circ} \mathrm{C}$, the stroke volume and CO fell. ${ }^{11}$ Our degree of cooling did not affect stroke volume.

To counteract hypothermia, healthy animals increase the rate of thermogenesis by a variety of mechanisms. Children, adults, and newborn animals without brown fat (such as the piglet) respond by shivering and restlessness with increased whole body oxygen consumption, cardiac contractility, and $\mathrm{CO}^{2}{ }^{22}$ This cardiovascular response is abolished by anaesthesia and heavy sedation. In full term newborn infants, non-shivering thermogenesis (brown fat oxidation) is the major route of a rapid increase in heat production in response to cold exposure. ${ }^{213}$

In our study we evaluated $\mathrm{CO}$ and recorded MABP and HR. We were not able to evaluate systemic vascular resistance or to assess ventricular function. The reported changes in CO could be due to changes in systemic vascular resistance, circulating volume, ductal patency, or ventricular function. We found no effect of ductal patency on CO or PAP. Circulating blood volume was not estimated, but we did not expect significant changes in the normothermic and hypothermic groups to occur when fluid treatment, haemoglobin, and blood sampling protocols were identical in the two groups.

The echocardiographic examinations did not show any morphological effects of hypothermia. That every third piglet had a PDA during one or more examinations is normal because piglets close their duct between 16 hours and five days after birth. ${ }^{14}$

Myocardial dysfunction, hypotension, and increased PAP with persistent pulmonary hypertension are well known consequences of hypoxic-ischaemic encephalopathy in the neonate, ${ }^{15}{ }^{16}$ with a relation between low alveolar oxygen tension and increased pulmonary vascular resistance in both animals and humans. ${ }^{17}$ These effects are found to be reversible for insults of short duration, in contrast with chronic hypoxia. ${ }^{18}$ The increase in PAP three hours after the insult reflects this transient increase in pulmonary vascular resistance, which is reversed with reoxygenation. We did not find in these piglets, which all had normal lungs and were ventilated with air, that 24 hours of hypothermia prolonged this increase in PAP.

Generalised hypothermia has physiological effects on virtually every organ system. Important effects are a generalised slowing of enzymatic activity and metabolic need. ${ }^{19}$ Cardiovascular responses to moderate hypothermia include peripheral vasoconstriction, ${ }^{20}$ sinus bradycardia with prolonged QT interval (reduced HR), and a decrease in CO and ejection fraction. ${ }^{21-23}$ In both animal experiments and human studies, these effects are dependent on the degree and duration of the hypothermia ${ }^{22} 23$; all these findings are from anaesthetised or sedated subjects. During mild hypothermia, however, there is evidence of both increased ${ }^{24}$ and reduced ${ }^{25}$ contractility. We found that the stroke volume did not change with mild hypothermia, therefore changes in CO were fully explained by changes in HR. We only found reduced CO in a subgroup of the hypothermic animals, namely those who suffered a long period ( $>30$ minutes) of low amplitude EEG. Most of these piglets also developed seizures, which is a marker of insult severity in our model.

In previous animal experiments and our clinical experience, we have occasionally observed hypotension during rewarming, which was probably due to vasodilatation. ${ }^{4}$ Since we started to routinely increase volume at the start of rewarming, hypotension has not been observed.

We did not find increased PAP in the hypothermic group compared with the normothermic group. Most of the early normal transition from fetal to neonatal pulmonary circulation had already taken place at the time of this experiment. In a pilot clinical study of hypothermia after birth asphyxia, cooling was associated with severe pulmonary hypertension in two of nine patients, which normalised when they were rewarmed. ${ }^{4}$ Pulmonary hypertension was, however, not a problem in the following 11 infants who were cooled (M Thoresen, unpublished work), and Shankaran et $a l^{16}$ did not find more pulmonary hypertension in the hypothermic group in their pilot study of total body cooling.

In conclusion, this animal study indicates that hypothermia after hypoxia has no adverse metabolic or cardiovascular effects. Mild hypothermia for 24 hours in unsedated newborn pigs after a severe hypoxic-ischaemic insult did not affect stroke volume or PAP when compared with normothermic pigs. A subgroup of hypothermic pigs that underwent the most severe hypoxic-ischaemic insult had reduced HR. The insult itself resulted in a transient increase in PAP, which was similar in both groups and unaffected by the severity of the insult, seizures, or whether the animals had an open ductus arteriosus. Twenty four hours of mild hypothermia did not affect the opening or closing of the ductus arteriosus.

\section{ACKNOWLEDGEMENTS}

The Laerdal Foundation for Acute Medicine, the Norwegian Research Council, and FUS Ullevål Hospital supported the study. We thank Fabio Apricena and Martin A Runde for excellent technical assistance, and Nycomed Imaging AS, Norway, for providing the ultrasound equipment.

\section{Authors' affiliations}

D Fugelseth, M Thoresen, Department of Paediatrics, Ullevål University Hospital, Oslo, Norway

D Fugelseth, S Satas, P A Steen, Institute for Experimental Medical Research, Ullevål University Hospital

S Satas, M Thoresen, Department of Child Health, St Michael's Hospital, University of Bristol, Bristol, UK

\section{REFERENCES}

1 Thoresen M. Cooling the newborn after asphyxia: physiological and experimental background and its clinical use. Semin Neonatol 2000;5:61-73.

2 Connor EL, Wren KR. Detrimental effects of hypothermia: a systems analysis. Journal of Perianesthesia Nursing 2000;15:151-5.

3 Thoresen M, Satas S, Løberg EM, et al. Twenty-four hours of mild hypothermia in unsedated newborn pigs starting after a severe global hypoxic-ischemic insult is not neuroprotective. Pediatr Res 2001;50:405-11.

4 Thoresen M, Whitelaw A. Cardiovascular changes during mild therapeutic hypothermia and rewarming in infants with hypoxic-ischemic encephalopathy. Pediatrics 2000;106:92-9.

5 Speidel B, Fleming P, Henderson J, et al. Care of the baby with respiratory distress. In: A neonatal vade mecum: neonatal respiratory problems. London: Edward Arnold, 1998:138-40.

6 Thoresen $M$, Haaland K, Løberg EM, et al. A piglet survival model of posthypoxic encephalopathy. Pediatr Res 1996;40:1-1 1

7 Fugelseth D, Satas S, Runde $M$, et al. Cardiac function and morphology studied by two-dimensional Doppler echocardiography in unsedated newborn pigs. Exp Physiol 1999;84:69-78.

8 Skjaerpe T, Hatle L. Diagnosis and assessment of tricuspid regurgitation with Doppler ultrasound. In: Rijsterborgh H, ed. Echocardiology. The Hague: Martinus Nijhoff, 1981:299-304.

9 Chan KL, Currie PJ, Seward JB, et al. Comparision of three Doppler ultrasound methods in the prediction of pulmonary artery pressure. J Am Coll Cardiol 1987;9:549-54.

10 Adams JA, Osiovich H, Goldberg, et al. Hemodynamic effects of continuous positive airway pressure in piglets with normal lungs. Biol Neonate 1992:62:69-75.

11 Chernow B, Lake CR, Zaritsky A, et al. Sympathetic nervous system "switch off" with severe hypothermia. Crit Care Med 1983;1 1:677-80.

12 Berthon D, Herpin P, Ledividich J. Shivering thermogenesis in the neonatal pig. J Therm Biol 1994;19:413-18.

13 Trayhurn P, Micholls DG, eds. Brown adipose tissue. London: Edward Arnold Ltd, 1986.

14 Evans JR, Phil D, Rowe RD. Murmurs arising from ductus arteriosus in normal newborn swine. Circ Res 1963;12:85-93.

15 Ranjit MS. Cardiac abnormalities in birth asphyxia. Indian J Pediatr 2000;67:529-32. 
16 Shankaran S, Laptook A, Wright LL, et al. Whole-body hypothermia for neonatal encephalopathy: animal observations as a basis for a randomized, controlled pilot study in term infants. Pediatrics 2002;110:377-85

17 Hambraeus-Jonzon K, Bindslev L, Mellgård Å, et al. Hypoxic pulmonary vasoconstriction in human lungs. Anesthesiology 1997:86:308-15.

18 Fike CD, Kaplowitz MR. Chronic hypoxia alters nitric oxide-dependent pulmonary vascular responses in lungs of newborn pigs. J Appl Physiol 1996;81:2078-87.

19 Rupp SM, Severinghaus JW. Hypothermia. In: Miller RD, ed. Anesthesia 2nd ed. New York: Churchill Livingstone, 1986:1995-2022.

20 Steen PA, Milde JH, Michenfelder JD. The detrimental effects of prolonged hypothermia and rewarming in the dog. Anesthesiology 1980;52:224-30.
21 Meyer DM, Horton JW. Effect of different degrees of hypothermia on myocardium in treatment of hemorrhagic shock. J Surg Res 1990;48:61-7.

22 Schubert A. Side effects of mild hypothermia. J Neurosurg Anesthesiol 1995; 7: 139-47.

23 Metz C, Holzschuh M, Bein T, et al. Moderate hypothermia in patients with severe head injury: cerebral and extracerebral effects. J Neurosurg 1996;85:533-41

24 Roscher R, Arlock P, Sjöberg T, et al. Effects of dopamine on porcine myocardial action potentials and contractions at $37^{\circ} \mathrm{C}$ and $32^{\circ} \mathrm{C}$. Acta Anaesthesiol Scand 2001:45:421-6.

25 Lewis ME, Al-Khalidi AH, Townend JN, et al. The effects of hypothermia on human left ventricular contractile function during cardiac surgery. Am Coll Cardiol 2002;39:102-8.

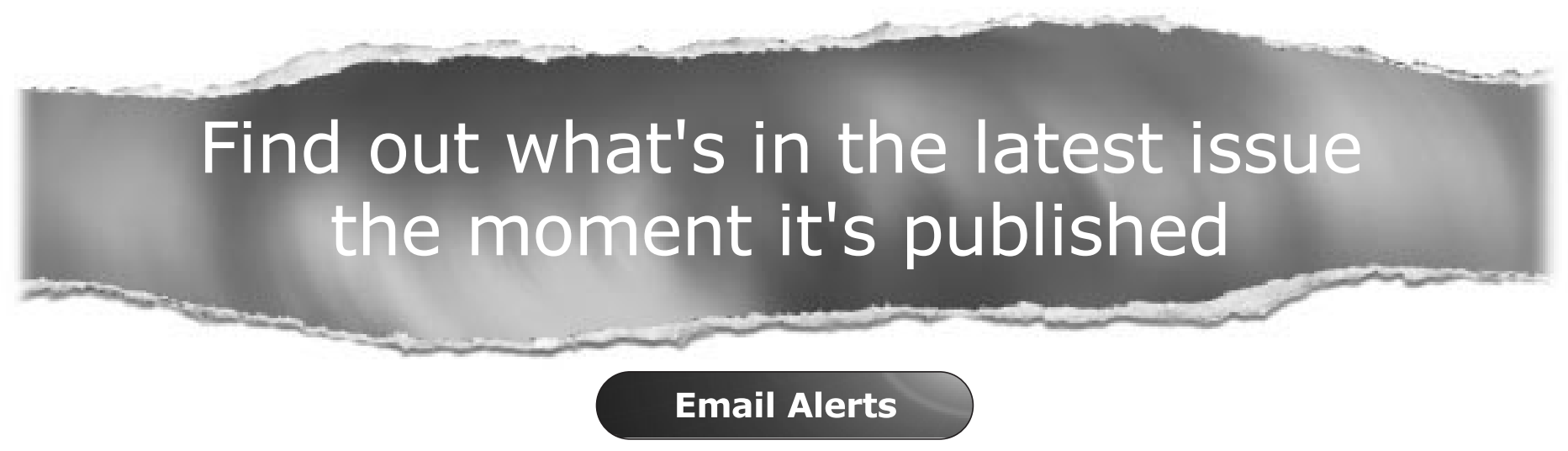

Sign up to receive the table of contents by email every month. You can select from three alerts: Table of Contents (full), TOC Awareness (notice only); Archives of Disease in Childhood related announcements.

www.archdischild.com 